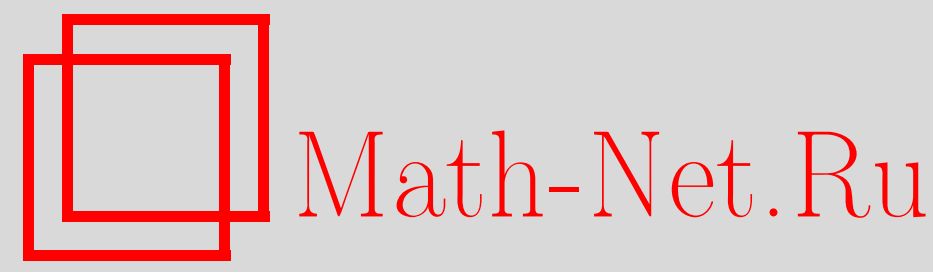

К. Н. Солтанов, Э. Б. Новрузов, Об одной задаче со свободной границей, Изв. РАН. Сер. матем., 2002, том 66, выпуск 4, 155-176

DOI: https://doi.org/10.4213/im398

Использование Общероссийского математического портала Math-Net.Ru подразумевает, что вы прочитали и согласны с пользовательским соглашением http://www . mathnet.ru/rus/agreement

Параметры загрузки:

IP : 54.224 .60 .19

26 апреля 2023 г., 10:59:04 
УДК 517.957

\author{
К.Н. Солтанов, Э.Б. Новрузов
}

\title{
Об одной задаче со свободной границей
}

\author{
Изучена гладкая разрешимость смешанной задачи для параболического урав- \\ нения с двойной нелинейностью. С использованием полученных результатов о \\ гладкости рассматриваемого уравнения исследована задача со свободной грани- \\ цей. \\ Библиография: 13 наименований.
}

\section{Введение}

Предметом настоящего исследования является одна задача со свободной границей (см. §3) для уравнения

$$
\frac{\partial u}{\partial t}-D\left(|u|^{p_{0}} D u+|D u|^{p_{1}} D u\right)=f(x, t) .
$$

Для этого изучаются гладкостные свойства решений уравнения (0.1) в некоторой области с фиксированной гранищей. Отметим, что необходимость получения гладкого решения уравнения является естественной при изучении задач со свободной границей. Получение такого решения еше более актуально в данном случае, поскольку для уравнения (0.1) преобразования, используемые, например, в работах $[1],[2]$ и позволяющие либо избавиться от нелинейности в главной части уравнения, либо свести задачу к некоторой вариационной проблеме, неприменимы. В частности, преобразование Кирхгофа (см. [1], [10]), позволяющее избавиться от нелинейности в главной части в случае уравнения с первым из нелинейных членов (0.1), в данном случае использовать невозможно.

Заметим, что задачам со свободной границей, в том числе и задаче Стефана для различных уравнений параболического типа, посвящено достаточно много работ (см., например, [2], [1]). В этих работах, в основном, рассмотрены либо линейные уравнения, либо уравнения, содержащие в главной части нелинейности, имеющие вид первого слагаемого в скобке уравнения (0.1). В некоторых работах указанные задачи для уравнений с подобного вида нелинейностями исследованы при достаточно общих условиях.

Определенные результаты о гладкости решений подобных уравнений содержатся, например, в работах [3], [4].

Отметим также, что вопросы разрешимости для уравнений такого вида, при довольно общих условиях на нелинейность, достаточно подробно исследованы в работах [5]-[7].

(C) К. Н. Солтанов, Э. Б. Новрузов, 2002 


\section{§1. Исследование начально-краевой задачи}

В этом параграфе при достаточно общих условиях доказано сушествование гладкого решения уравнения (0.1) в цилиндрической области, благодаря чему, в частности, можно сделать заключение о гладкости гиперповерхности $u(x, t)=0$. В дальнейшем можно использовать полученный результат при исследовании свойств свободной границы.

Рассмотрим следующую задачу:

$$
\begin{gathered}
\frac{\partial u}{\partial t}-D\left(|u|^{p_{0}} D u+|D u|^{p_{1}} D u\right)=f(x, t), \quad(x, t) \in Q \equiv(0, T) \times \Omega, \\
u(x, 0)=u_{0}(x), \quad x \in \Omega \equiv(a, b), \\
\left.u\right|_{\Gamma}=0, \quad \Gamma \equiv \partial \Omega \times[0, T),
\end{gathered}
$$

где $f(x, t), u_{0}(x)$ - некоторые функции, $D=\frac{\partial}{\partial x}, p_{0} \geqslant 2, p_{1} \geqslant 1,(a, b)$ - ограниченный интервал.

Введем следующее пересечение пространств функций $u: Q \rightarrow \mathbb{R}$ :

$$
\begin{aligned}
& \Re_{1}(Q) \equiv L_{\nu}\left(0, T ; \stackrel{\circ}{S}_{1, p_{0}+p_{*}, 2}(\Omega)\right) \cap L_{\infty}\left(0, T ; \stackrel{\circ}{W}_{\aleph+2}^{1}(\Omega)\right) \cap L_{\mu}\left(0, T ; \stackrel{\circ}{S}_{1, p_{*}, p_{1}+2}(\Omega)\right) \cap \\
& \cap L_{\nu_{1}}\left(0, T ; S_{\circ}^{1}{ }_{1, p_{1}+\aleph, 2}(\Omega)\right) \cap W_{2}^{1}(Q) \cap L_{p_{0}+2}\left(0, T ; \stackrel{\circ}{S}_{2, p_{0}, 2}(\Omega)\right) \cap \\
& \cap L_{p_{1}+2}\left(0, T ; S_{1, p_{1}, 2}^{1}(\Omega)\right) \cap\left\{u \mid u(x, 0)=u_{0}(x)\right\}, \\
& \stackrel{\circ}{S}_{1, \beta, \gamma}(\Omega)=\left\{\left.u(x)\left|\int_{\Omega}\right| u\right|^{\beta}|D u|^{\gamma} d x<+\infty,\left.u\right|_{\partial \Omega}=0\right\}, \\
& \stackrel{\circ}{W}_{\gamma}^{1}(\Omega)=\left\{u(x)\left|\left(\int_{\Omega}|u(x)|^{\gamma}+|D u|^{\gamma} d x\right)^{\frac{1}{\gamma}}<+\infty, u\right|_{\partial \Omega}=0\right\},
\end{aligned}
$$

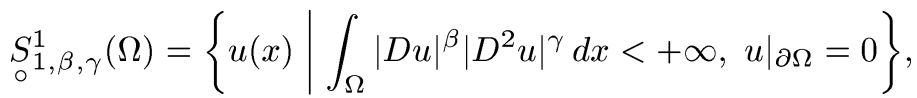

$$
\begin{aligned}
& \stackrel{\circ}{S}_{2, \beta, \gamma}(\Omega)=\left\{\left.u(x)\left|\int_{\Omega}\right| u\right|^{\beta}\left|D^{2} u\right|^{\gamma} d x<+\infty,\left.u\right|_{\partial \Omega}=0\right\}
\end{aligned}
$$

(о пространствах $S_{\alpha, \beta, \gamma}^{k}$ см. [8]).

ОПРЕДЕЛЕНИЕ 1.1. Решением задачи (1.1)-(1.3) назовем функцию $u(x, t) \in$ $\Re_{1}(Q)$, удовлетворяюшую уравнению (1.1) в смысле пространства $L_{2}(Q)$, т.е. для любого $v(x, t) \in L_{2}(Q)$ выполняется равенство

$$
\int_{Q} \frac{\partial u}{\partial t} v d x d t-\int_{Q} D\left(|u|^{p_{0}} D u+|D u|^{p_{1}} D u\right) v d x d t=\int_{Q} f v d x d t
$$


Теорема 1.1. Пусть функиии и $u_{0}, f$ удовлетворяют следующим условиям:

$$
u_{0} \in \stackrel{\circ}{W}_{\aleph+2}^{1}(\Omega) \cap L_{p_{*}+2}(\Omega), \quad f \in L_{2}(0, T ; \stackrel{\circ}{W} \underset{\aleph+2}{1}(\Omega)), \quad p_{1} \geqslant 1, \quad p_{0} \geqslant 2 .
$$

Тогда задача (1.1)-(1.3) разрешима в смысле определения (более того, п.в. в $Q)$.

Для доказательства этой теоремы будет использован обобщенный метод компактности [5]. Соответствуюшие априорные оценки будут получены с применением неравенства обобщенной коэрцитивности, справедливость которого, в свою очередь, доказывается с помощью построения некоторого оператора, порождаюшего коэрцитивную пару с оператором, порожденным рассмотренной задачей.

Остановимся теперь на построении упомянутого оператора. Введем следуюшие пространства:

$$
\begin{aligned}
& \Re_{2}(Q) \equiv \Re_{1}(Q) \cap L_{2}\left(0, T ; W_{2}^{2}(\Omega)\right) \cap L_{\aleph+2}\left(0, T ; S_{\circ}^{1}{ }_{\circ, \aleph, 2}(\Omega)\right) \cap \\
& \cap\left\{\left.v|| D v\right|^{\aleph / 2} D v_{t} \in L_{2}(Q)\right\} \cap\left\{v \mid D v_{t} \in L_{2}(Q)\right\}, \\
& \Re_{2}^{\prime}(Q) \equiv \Re_{1}(Q) \cap L_{2}\left(0, T ; W_{2}^{2}(\Omega)\right) \cap L_{\aleph+2}\left(0, T ; S_{0}^{1} 1_{, \aleph 2}(\Omega)\right) \cap \\
& \cap\left\{\left.v|\sqrt{T-t}| D v\right|^{\aleph / 2} D v_{t} \in L_{2}(Q)\right\} \cap\left\{v \mid \sqrt{T-t} D v_{t} \in L_{2}(Q)\right\} .
\end{aligned}
$$

Пусть $E: \Re_{2}(Q) \rightarrow W_{2}^{1}(Q) \subset L_{2}(Q)$ - сужение на $Q$ оператора $\widetilde{E}: \Re_{2}^{\prime}\left(Q_{1}\right) \rightarrow$ $W_{2}^{1}\left(Q_{1}\right)$, порожденного задачей

$$
\begin{gathered}
c_{2} u_{t}-|D u|^{\aleph} D^{2} u-c_{3} D^{2} u-c_{1}|u|^{p_{*}} u=h(x, t), \quad(x, t) \in Q_{1} \equiv(a, b) \times\left(0, T_{1}\right), \\
u(x, 0)=u_{0}(x), \quad x \in \Omega, \\
\left.u\right|_{\Gamma_{1}}=0, \quad \Gamma_{1} \equiv \partial \Omega \times\left[0, T_{1}\right),
\end{gathered}
$$

где $T_{1}>T ; \aleph, p_{*}, c_{1}, c_{2}, c_{3}$ - положительные постоянные.

TEOPEMA 1.2. Пусть $h(x, t) \in W_{2}^{1}\left(Q_{1}\right), \quad Q_{1} \equiv \Omega \times\left[0, T_{1}\right)$. Тогда при любых $\aleph>0$ и $u_{0}(x) \in W_{2}^{1}(\Omega) \cap S_{1, \aleph, 2}^{1}(\Omega) \cap L_{p_{*}+2}(\Omega)$ существует, причем единственное, решение $u(x, t) \in \Re_{2}^{\prime}\left(Q_{1}\right)$ задачи (1.4)-(1.6) в смысле, аналогичном определению 1.1.

ДоКАЗАТЕЛЬСТВо аналогично доказательству соответствующей теоремы из [9], где задача (1.4)-(1.6) исследована в случае, когда $c_{2}=1, c_{3}=c_{1}=0$. Доказательство, предложенное в [9], позволяет сделать вывод о разрешимости задачи (1.4)-(1.6) в случае произвольных $c_{1}, c_{2}, c_{3}>0$.

В дальнейшем будем считать, что $c_{3}=1$. Таким образом, можно определить оператор $E: \Re_{2}(Q) \rightarrow \stackrel{\circ}{W} \frac{1}{2}(Q)$, для которого справедлива следуюшая лемма.

Лемма 1.1. Оператор $E^{-1}: \stackrel{\circ}{W} \underset{2}{1}(Q) \rightarrow \Re_{2}(Q)$ слабо компактен. 
ДоКАЗАТЕЛЬСТВО. Пусть $\left\{h_{n}\right\}$ - слабо сходящаяся последовательность из $\stackrel{\circ}{W} \frac{1}{2}(Q)$, т.е. $h_{n} \rightarrow h_{0}$. Пусть $u_{n}-$ прообраз элемента $h_{n}$. Он существует и единствен в силу теоремы 1.2 . Далее, $E\left(u_{n}\right)=h_{n} \rightarrow h_{0}=E(u)$. Естественно, что последовательность $u_{n}$ равномерно ограничена в $\Re_{2}(Q)$. Покажем, что $u_{n} \rightarrow u$ слабо (может быть, после перехода на подпоследовательность).

В самом деле, так как последовательность $\left\{u_{n}\right\}$ равномерно ограничена в $\Re_{2}(Q)$, то из нее можно выделить подпоследовательность $\left\{u_{n k}\right\}$, сходящуюся к некоторому $v \in \Re_{2}(Q)$. Следовательно, $E\left(u_{n k}\right) \rightarrow E(v)$. Но $E\left(u_{n k}\right) \rightarrow h_{0}=E(u)$. Иначе говоря, $E(v)=E(u)$. Отсюда в силу однозначной разрешимости задачи $(1.4)-(1.6)$ получаем, что $v=u$. Таким образом, $u_{n k} \rightarrow u$ слабо в $\Re_{2}(Q)$, что и требовалось доказать.

Введем теперь следуюшие обозначения:

$$
\begin{gathered}
\psi(u)=\frac{\partial u}{\partial t}-\left(D|u|^{p_{0}} D u\right)-D\left(|u|^{p_{1}} D u\right), \\
A(u)=-D\left(|D u|^{p_{1}} D u\right), \\
B(u)=-D\left(|u|^{p_{0}} D u\right) .
\end{gathered}
$$

Докажем, что операторы $A: \Re_{1}(Q) \rightarrow L_{2}(Q)$ и $B: \Re_{1}(Q) \rightarrow L_{2}(Q)$ являются ограниченными.

Действительно, применяя неравенства Юнга и Гёльдера для любого $u \in \Re_{1}(Q)$, имеем

$$
\begin{aligned}
\|A(u)\|_{L_{2}(Q)}^{2} & =\left(p_{1}+1\right)\left\||D u|^{p_{1}} D^{2} u\right\|_{L_{2}(Q)}^{2}=\left(p_{1}+1\right) \int_{Q}|D u|^{2 p_{1}}\left|D^{2} u\right|^{2} d x d t \\
& \leqslant\left(p_{1}+1\right) \int_{Q}|D u|^{p_{1}+\aleph}\left|D^{2} u\right|^{2} d x d t+\left(p_{1}+1\right) \int_{Q}|D u|^{p_{1}}\left|D^{2} u\right|^{2} d x d t \\
& =\left(p_{1}+1\right)\left([u]_{L_{\nu_{1}}\left(0, T ; S_{1, p_{1}+\aleph, 2}^{1}(\Omega)\right)}+[u]_{L_{p_{1}+2}\left(0, T ; S_{1, p_{1}, 2}^{1}(\Omega)\right)}\right) .
\end{aligned}
$$

Аналогично доказывается ограниченность оператора $B: \Re_{1}(Q) \rightarrow L_{2}(Q)$.

Докажем теперь, учитывая сказанное вьше, следуюшую лемму.

Лемма 1.2. Оператор $\psi: \Re_{1}(Q) \rightarrow L_{2}(Q)$, порожденный задачей (1.1)(1.3), является слабо компактным.

ДОКАЗАТЕЛЬСТВО. Пусть $u_{m}$ - ограниченная последовательность из пространства $\Re_{1}(Q)$. "Рефлексивность" данного пространства показывает, что из последовательности $u_{m}$ можно выделить слабо сходящуюся подпоследовательность. Кроме того, ввиду ограниченности операторов $A$ и $B$ и ввиду рефлексивности $L_{2}(Q)$ получаем, что из последовательностей $A\left(u_{m}\right)$ и $B\left(u_{m}\right)$ можно выделить слабо сходяшиеся подпоследовательности.

Итак, при $m_{k} \nearrow \infty$ имеем $A\left(u_{m_{k}}\right) \rightarrow \eta, B\left(u_{m_{k}}\right) \rightarrow \chi$ в $L_{2}(Q)$, т.е. имеет место соотношение: $A\left(u_{m_{k}}\right)+B\left(u_{m_{k}}\right) \rightarrow \eta+\chi$ слабо в $L_{2}(Q)$. Так как $\Re_{1}(Q)$ вложено в

$$
L_{2 p_{0}+2}\left(0, T ; \stackrel{\circ}{S}_{1,2 p_{0}, 2}(\Omega)\right) \cap L_{2 p_{1}+2}\left(0, T ; \stackrel{\circ}{W}_{2 p_{1}+2}^{1}(\Omega)\right)
$$


и вложено компактно (см. [8]), имеем

$$
\begin{array}{ll}
u_{m_{k}} \rightarrow u & \text { в } L_{p_{0}+2}\left(0, T ; \stackrel{\circ}{S}_{1, p_{0}, 2}(\Omega)\right), \\
\left|u_{m_{k}}\right|{ }^{p_{0}} D u_{m_{k}} \rightarrow|u|^{p_{0}} D u & \text { в } \quad L_{2}(Q), \\
D u_{m_{k}} \rightarrow D u & \text { в } L_{2}(Q), \\
\left|D u_{m_{k}}\right|^{p_{1}} D u_{m_{k}} \rightarrow|D u|^{p_{1}} D u & \text { в } \quad L_{2}(Q) .
\end{array}
$$

Используя эти соотношения, легко показать, что $A: \Re_{1}(Q) \rightarrow L_{2}(Q)$ и $B$ : $\Re_{1}(Q) \rightarrow L_{2}(Q)$ - слабо компактные операторы.

Покажем, что $B: \Re_{1}(Q) \rightarrow L_{2}(Q)$ является слабо компактным оператором. Действительно, для любого элемента $v \in L_{p_{0}+2}\left(0, T ; \stackrel{\circ}{W} \underset{p_{0}+2}{1}(\Omega)\right)$

$$
\begin{aligned}
\int_{Q} B\left(u_{m_{k}}\right) v d x d t & =-\int_{Q} D\left(\left|u_{m_{k}}\right|{ }^{p_{0}} D u_{m_{k}}\right) v d x d t=\int_{Q}\left|u_{m_{k}}\right|{ }^{p_{0}} D u_{m_{k}} D v d x d t \rightarrow \\
& \rightarrow \int_{Q}|u|^{p_{0}} D u D v d x d t=-\int D\left(|u|^{p_{0}} D u\right) v d x d t .
\end{aligned}
$$

Следовательно, $B u=\eta$ в $L_{q_{0}}\left(0, T ; W_{q_{0}}^{-1}(\Omega)\right)$, где $q_{0}=\frac{p_{0}+2}{p_{0}+1}, q_{0} \leqslant 2$. Так как в силу вышеизложенного $B\left(u_{m_{k}}\right) \rightarrow \eta$ в $L_{2}(Q)$, то $B\left(u_{m_{k}}\right) \rightarrow B u$ в $L_{2}(Q)$.

Итак, $B: \Re_{1}(Q) \rightarrow L_{2}(Q)$ - слабо компактный оператор.

Аналогично доказывается слабая компактность оператора $A$. Слабая компактность оператора $\frac{\partial}{\partial t}: \Re_{1}(Q) \rightarrow L_{2}(Q)$ вытекает из его линейности.

Следовательно, оператор $\psi=\left(\frac{\partial}{\partial t}+A+B\right), \psi: \Re_{1}(Q) \rightarrow L_{2}(Q)$, слабо компактен, что и требовалось доказать.

ДОКАЗАТЕЛЬСТвО ТЕОРЕмЫ 1.1. Пусть $\left\{y_{k}\right\}$ - полная система в пространстве $W_{2}^{1}(Q)$. Приближенное решение уравнения (1.1) находим в виде

$$
u_{m}=E^{-1}\left(y_{m}^{*}\right), \quad y_{m}^{*}=\sum_{k=1}^{m} c_{m k} y^{k}
$$

при этом неизвестные коэффициенты $c_{m k}$ находятся из системы алгебраических уравнений

$$
\Phi_{k}\left(c_{m}\right) \equiv\left\langle\psi\left(u_{m}\right), y^{k}\right\rangle-\left\langle f, y^{k}\right\rangle=0, \quad k=1,2, \ldots, m
$$

$\left(\langle\cdot, \cdot\rangle\right.$ - двойственная форма для пары $\left(X, X^{*}\right)$; в данном случае $\left.X=L_{2}(Q)\right)$. Разрешимость системы (1.9) вытекает из известной леммы об остром угле.

ЛЕмма (об остром угле) [10]. Пусть $\xi \rightarrow P(\xi)-$ такое непрерывное отображение $\mathbb{R}^{m}$ в себя, что для подходящего $\rho>0$

$$
(P(\xi), \xi) \geqslant 0 \quad \forall \xi \quad \text { из сферьл }|\xi|=\rho,
$$


где для $\xi=\left\{\xi_{1}, \ldots, \xi_{m}\right\} \in \mathbb{R}^{m}$ u $\eta=\left\{\eta_{1}, \ldots, \eta_{m}\right\} \in \mathbb{R}^{m}$ полагаем, ито

$$
(\xi, \eta)=\sum_{k=1}^{m} \xi_{k} \eta_{k}, \quad|\xi|=(\xi, \xi)^{1 / 2}
$$

Тогда найдется такое $\xi,|\xi| \leqslant \rho$, что $P(\xi)=0$.

В силу введенных обозначений имеем

$$
\begin{gathered}
\Phi_{k}\left(c_{m}\right) \equiv\left\langle\psi\left(u_{m}\right), y^{k}\right\rangle-\left\langle f, y^{k}\right\rangle, \quad \xi=\left\{\xi_{1}, \ldots, \xi_{m}\right\}=\left\{c_{m 1}, \ldots, c_{m m}\right\}=c_{m} \\
P(\xi)=\Phi\left(c_{m}\right)
\end{gathered}
$$

Тогда

$$
(P(\xi), \xi)=\left(\Phi\left(c_{m}\right), c_{m}\right)=\sum_{k=1}^{m} c_{m k} \Phi_{k}\left(c_{m}\right)=\left\langle\psi\left(u_{m}\right), E\left(u_{m}\right)\right\rangle-\left\langle f, E\left(u_{m}\right)\right\rangle
$$

Заметим, во-первых, что из слабой непрерывности операторов $E^{-1}$ и $\psi$ вытекает непрерывность отображения $\Phi$ из $\mathbb{R}^{m}$ в $\mathbb{R}^{m}$. Поэтому остается проверить, что сушествует $\rho$ такое, что для любого $\xi$ из сферы $|\xi|=\rho$

$$
\left\langle\psi\left(u_{m}\right), E\left(u_{m}\right)\right\rangle-\left\langle f, u_{m}\right\rangle \geqslant 0 .
$$

Проверим этот факт.

ЛЕмма 1.3. Пусть $u_{m}(x, t)$ удовлетворяет условию (1.3), $u_{m}(x, t) \in \Re_{2}(Q)$. Тогда справедливо следующее равенство:

$$
\begin{aligned}
& \left.\left\langle\psi\left(u_{m}\right), c_{1}\left|u_{m}\right|^{p_{*}} u_{m}\right\rangle\right|_{\tau}=\left.\frac{c_{1}}{p_{*}+2} \int_{\Omega}\left|u_{m}\right|^{p_{*}+2} d x\right|_{\tau} \\
& \quad+c_{1}\left(p_{*}+1\right) \int_{Q_{\tau}}\left|u_{m}\right|^{p_{*}+p_{0}}\left(D u_{m}\right)^{2} d x d t \\
& \quad+c_{1}\left(p_{*}+1\right) \int_{Q_{r}}\left|u_{m}\right|^{p_{*}}\left|D u_{m}\right|^{p_{1}+2} d x d t-\frac{c_{1}}{p_{*}+2} \int_{\Omega}\left|u_{0}\right|^{p_{*}+2} d x
\end{aligned}
$$

əде $Q_{\tau}=[0, \tau] \times \Omega, \quad p_{*}=\left(4 p_{0}-2\right)(\aleph+2), \quad c_{1}>0$.

Доказательство непосредственно следует из формулы интегрирования по частям. 
Лемма 1.4. Пусть $u_{m}(x, t)$ удовлетворяет условию $(1.3), u_{m}(x, t) \in \Re_{2}(Q)$. Тогда справедливо следующее неравенство:

$$
\begin{aligned}
& \left.\left\langle\psi\left(u_{m}\right),-D^{2} u_{m}\right\rangle\right|_{\tau} \geqslant\left.\frac{1}{2} \int_{\Omega}\left|D u_{m}\right|^{2} d x\right|_{\tau}+\int_{Q_{r}}\left|u_{m}\right|^{p_{0}}\left|D^{2} u_{m}\right|^{2} d x d t \\
& \quad+M_{1} \int_{Q_{r}}\left|D u_{m}\right|^{p_{1}}\left|D^{2} u_{m}\right|^{2} d x d t-\frac{1}{2} \int_{\Omega}\left|D u_{0}\right|^{2} d x \\
& -M_{2} \int_{Q_{r}}\left|u_{m}\right|^{p_{1}^{*}}\left|D u_{m}\right|^{p_{1}+2} d x d t-M_{3} \int_{Q_{r}}\left|u_{m}\right|^{p_{2}^{*}} d x d t,
\end{aligned}
$$

причем:

а) если числа $p_{0}$ и $p_{1}$, удовлетворяют условиям $p_{0} \geqslant 2, \frac{1}{2} \leqslant p_{1} \leqslant 4$, то

$$
\begin{aligned}
M_{1}=p_{1}+\frac{2}{3}, \quad M_{2} & =\frac{3 p_{0}^{2}}{2} \frac{\left(p_{1}+2\right)}{\left(2 p_{0}-1\right)} \times \frac{9 p_{0}^{2}}{4} \frac{\left(p_{1}+2\right)}{\left(2 p_{0}-1\right)}, \quad M_{3}=\frac{3 p_{0}^{2}}{2} \\
p_{1}^{*} & =4 p_{0}-2, \quad p_{2}^{*}=2 p_{0}-2 ;
\end{aligned}
$$

б) $е с л и p_{0} \geqslant 2, p_{1}>4, m o$

$$
M_{1}=p_{1}+1, \quad M_{2}=M_{3}=\frac{p_{0}\left(p_{0}-1\right)}{3}, \quad p_{1}^{*}=p_{2}^{*}=p_{0}-2 .
$$

ДоКАЗАТЕльСТво. Для любого $u_{m} \in \Re_{2}\left(Q_{\tau}\right)$ справедливо тождество

$$
\begin{aligned}
& \left.\left\langle\psi\left(u_{m}\right),-D^{2} u_{m}\right\rangle\right|_{\tau}=-\int_{Q_{r}} u_{m t} D^{2} u_{m} d x d t+\int_{Q_{r}}\left|u_{m}\right|^{p_{0}}\left|D^{2} u_{m}\right|^{2} d x d t \\
& \quad+\left(p_{1}+1\right) \int_{Q_{r}}\left|D u_{m}\right|^{p_{1}}\left|D^{2} u_{m}\right|^{2} d x d t+p_{0} \int_{\Omega}\left|u_{m}\right|^{p_{0}-2} u_{m}\left|D u_{m}\right|^{2} D^{2} u_{m} d x d t
\end{aligned}
$$

Заметим, что

$$
-\int_{Q_{r}} u_{m t} D^{2} u_{m} d x d t=\int_{Q_{r}} D u_{m} D u_{m t} d x d t=\frac{1}{2} \int_{Q_{r}} \frac{\partial}{\partial t}\left|D u_{m}\right|^{2} d x d t,
$$

так как из равенства $\left.u_{m}\right|_{\Gamma}=0$ вытекает, что $\left.u_{m t}\right|_{\Gamma}=0$, поскольку в классе рассматриваемых функций гладкие функции всюду плотны.

Далее рассмотрим следующие случаи.

I. Пусть $p_{0}>1, \frac{1}{2} \leqslant p_{1} \leqslant 4$. Докажем справедливость следующего неравенства:

$$
\begin{aligned}
\int_{Q_{r}}\left|u_{m}\right|^{p_{*}}\left|D u_{m}\right|^{p_{1}+3} d x d t \leqslant & c(\varepsilon) \int_{Q_{r}} \frac{p_{1}+2}{p_{*}+1}\left|u_{m}\right|^{2 p_{*}+2}\left|D u_{m}\right|^{p_{1}+2} d x d t \\
& +\varepsilon \frac{p_{1}+2}{p_{*}+1} \int_{Q_{r}}\left|D u_{m}\right|^{p_{1}}\left|D^{2} u_{m}\right|^{2} d x d t
\end{aligned}
$$


где $p_{*}>0, \varepsilon \succ 0-$ произвольные постоянная.

Действительно, используя интегрирование по частям и неравенство Юнга, получаем

$$
\begin{aligned}
& \left.\left|\int_{Q_{r}}\right| u_{m}\right|^{p_{*}}\left|D u_{m}\right|^{p_{1}+1} D u_{m} D u_{m} d x d t \mid \leqslant \\
& \leqslant c(\varepsilon) \frac{p_{1}+2}{p_{*}+1} \int_{Q_{r}}\left|u_{m}\right|^{2 p_{*}+2}\left|D u_{m}\right|^{p_{1}+2} d x d t \\
& \quad+\varepsilon \frac{p_{1}+2}{p_{*}+1} \int_{Q_{r}}\left|D u_{m}\right|^{p_{1}}\left|D^{2} u_{m}\right|^{2} d x d t
\end{aligned}
$$

Теперь, применяя к интегралу $\int_{Q_{r}}\left|u_{m}\right|^{p_{0}-2} u_{m}\left|D u_{m}\right|^{2} D^{2} u_{m} d x d t$ неравенство Юнга, имеем

$$
\begin{aligned}
& \left.\left|\int_{Q_{r}}\right| u_{m}\right|^{p_{0}-2} u_{m}\left|D u_{m}\right|^{2} D^{2} u_{m} d x d t \mid \\
& =\left.\left|\int_{Q_{r}}\right| u_{m}\right|^{p_{0}-2} u_{m}\left|D u_{m}\right|^{2-\frac{p_{1}}{2}}\left|D u_{m}\right|^{\frac{p_{1}}{2}} D^{2} u_{m} d x d t \mid \\
& \leqslant c\left(\varepsilon_{1}\right) \int_{Q_{r}}\left|u_{m}\right|^{2 p_{0}-2}\left|D u_{m}\right|^{4-p_{1}} d x d t+\varepsilon_{1} \int_{Q_{r}}\left|D u_{m}\right|^{p_{1}}\left|D^{2} u_{m}\right|^{2} d x d t \\
& \leqslant c\left(\varepsilon_{1}\right) \int_{Q_{r}}\left|u_{m}\right|^{2 p_{0}-2}\left|D u_{m}\right|^{p_{1}+3} d x d t+c\left(\varepsilon_{1}\right) \int_{Q_{r}}\left|u_{m}\right|^{2 p_{0}-2} d x d t \\
& +\varepsilon_{1} \int_{Q_{r}}\left|D u_{m}\right|^{p_{1}}\left|D^{2} u_{m}\right|^{2} d x d t
\end{aligned}
$$

Последний переход следует из очевидного неравенства $|a|^{4-p_{1}} \leqslant a^{p_{1}+3}+1$, справедливого для любого $a \geqslant 0$, при условии $4-p_{1} \leqslant p_{1}+3$, которое эквивалентно предположению, что $p_{1} \geqslant \frac{1}{2}$.

Применяя неравенство (1.12) при $p_{*}=2 p_{0}-2$, окончательно имеем

$$
\begin{aligned}
& \left.\left.p_{0}\left|\int_{Q_{r}}\right| u_{m}\right|^{p_{0}-2} u_{m}\left|D u_{m}\right|^{2} D^{2} u_{m} d x d t\left|\leqslant p_{0} c\left(\varepsilon_{1}\right) \int_{Q_{r}}\right| u_{m}\right|^{2 p_{0}-2} d x d t \\
& +p_{0} \varepsilon_{1} \int_{Q_{r}}\left|D u_{m}\right|^{p_{1}}\left|D^{2} u_{m}\right|^{2} d x d t \\
& +p_{0} c\left(\varepsilon_{1}\right) \frac{p_{1}+2}{2 p_{0}-1} c(\varepsilon) \int_{Q_{r}}\left|u_{m}\right|^{2\left(2 p_{0}-2\right)+2}\left|D u_{m}\right|^{p_{1}+2} d x d t \\
& +p_{0} \varepsilon \frac{p_{1}+2}{2 p_{0}-1} c\left(\varepsilon_{1}\right) \int_{Q_{r}}\left|D u_{m}\right|^{p_{1}}\left|D^{2} u_{m}\right|^{2} d x d t \\
& =p_{0} c\left(\varepsilon_{1}\right) c(\varepsilon) \frac{p_{1}+2}{2 p_{0}-1} \int_{Q_{r}}\left|u_{m}\right|^{4 p_{0}-2}\left|D u_{m}\right|^{p_{1}+2} d x d t \\
& +\left(p_{0} c\left(\varepsilon_{1}\right) \varepsilon \frac{p_{1}+2}{2 p_{0}-1}+p_{0} \varepsilon_{1}\right) \int_{Q_{r}}\left|D u_{m}\right|^{p_{1}}\left|D^{2} u_{m}\right|^{2} d x d t \\
& +p_{0} c\left(\varepsilon_{1}\right) \int_{Q_{r}}\left|u_{m}\right|^{2 p_{0}-2} d x d t
\end{aligned}
$$


Далее, выбирая $\varepsilon$ и $\varepsilon_{1}$ соответствуюшим образом и возврашаясь к равенствам (1.10) и (1.11), получаем искомьй результат.

II. Пусть теперь $p_{1} \geqslant 4, p_{0} \geqslant 2$. Тогда имеем

$$
\begin{gathered}
\left.p_{0}\left|\int_{Q_{r}}\right| u_{m}||^{p_{0}-2}\left|D u_{m}\right|^{2} D^{2} u_{m} d x d t\left|=\frac{\left(p_{0}-1\right) p_{0}}{3} \int_{Q_{r}}\right| u_{m}\right|^{p_{0}-2}\left|D u_{m}\right|^{4} d x d t \\
\leqslant \frac{\left(p_{0}-1\right) p_{0}}{3}\left(\int_{Q_{r}}\left|u_{m}\right|^{p_{0}+2}\left|D u_{m}\right|^{p_{1}+2} d x d t+\int_{Q_{r}}\left|u_{m}\right|^{p_{0}-2} d x d t\right) .
\end{gathered}
$$

Учитывая последнее неравенство в (1.10), получаем справедливость неравенства леммы 1.4. Лемма полностью доказана.

Лемма 1.5. Пусть $u_{m}(x, t)$ удовлетворяет условиям леммы 1.4. Тогда справедливо следующее неравенство:

$$
\begin{aligned}
\left\langle\psi\left(u_{m}\right),-\right. & \left.\left|D u_{m}\right|^{\aleph} D^{2} u_{m}\right\rangle\left.\right|_{\tau} \geqslant \frac{1}{\aleph+2} \int_{\Omega}\left|D u_{m}(x, \tau)\right|^{\aleph+2} d x \\
& +\int_{Q_{r}}\left|u_{m}\right|^{p_{0}}\left|D u_{m}\right|^{\aleph}\left|D^{2} u_{m}\right|^{2} d x d t \\
& +\left(p_{1}+\frac{1}{3}\right) \int_{Q_{r}}\left|D u_{m}\right|^{p_{1}+\aleph}\left|D^{2} u_{m}\right|^{2} d x d t \\
& -\frac{1}{\aleph+2} \int_{\Omega}\left|D u_{m}(x, 0)\right|^{\aleph+2} d x-\frac{2}{3} \int_{Q_{r}}\left|D u_{m}\right|^{p_{1}}\left|D^{2} u_{m}\right|^{2} d x d t \\
& -c_{1}^{*} \int_{Q_{r}}\left|u_{m}\right|^{\aleph+2} d x d t-c_{2}^{*} \int_{Q_{r}}\left|u_{m}\right|^{\left(4 p_{0}-2\right)(\aleph+2)} d x d t,
\end{aligned}
$$

где $\geqslant p_{1}, p_{1} \geqslant 1, p_{0}>1, c_{1}^{*}, c_{2}^{*}$ - некоторые положительные постоянные.

Вся сложность доказательства леммы 1.5 заключается в получении оценки, которая следует из неравенства Юнга:

$$
\begin{aligned}
\left|\int_{Q_{r}}\right| u_{m}||^{p_{0}-2} u_{m}\left|D u_{m}\right|^{\aleph+2} D^{2} u_{m} d x d t \mid \\
\quad \leqslant \frac{1}{2}\left(\frac{\varepsilon_{1}^{2}(\aleph+2)}{2 p_{0}-1}+1\right) \int_{Q_{r}}\left|D u_{m}\right|^{p_{1}}\left|D^{2} u_{m}\right|^{2} d x d t \\
\quad+\frac{1}{2}\left(\frac{\varepsilon_{1}^{2}(\aleph+2)}{2 p_{0}-1}+1\right) \int_{Q_{r}}\left|D u_{m}\right|^{p_{1}+\aleph}\left|D^{2} u_{m}\right|^{2} d x d t \\
\quad+\frac{(\aleph+1)}{2 p_{0}-1} \varepsilon_{2} \cdot \frac{1}{2 \varepsilon_{1}^{2}} \int_{\Omega}\left|D u_{m}\right|^{\aleph+2} d x \\
\quad+\frac{1}{2 \varepsilon_{1}^{2}\left(2 p_{0}-1\right) \varepsilon_{2}^{\aleph+1}} \int_{Q_{r}}\left|u_{m}\right|^{\left(4 p_{0}-2\right)(\aleph+2)} d x d t,
\end{aligned}
$$

где $\varepsilon_{1} \succ 0, \varepsilon_{2} \succ 0$ - достаточно малые величины. 
Далее, легко можно показать, что (см. [8])

$$
\begin{aligned}
\int_{Q_{\tau}}\left|D u_{m}\right|^{\aleph+2} d x d t \leqslant \int_{Q_{\tau}}\left|D u_{m}\right|^{\aleph}\left|D^{2} u_{m}\right|^{2} d x d t+c^{*} \int_{Q_{\tau}}\left|u_{m}\right|^{\aleph+2} d x d t \\
\leqslant \int_{Q_{\tau}}\left|D u_{m}\right|^{\aleph+p_{0}}\left|D^{2} u_{m}\right|^{2} d x d t+\int_{Q_{\tau}}\left|D u_{m}\right|^{p_{0}}\left|D^{2} u_{m}\right|^{2} d x d t \\
\quad+c^{*} \int_{Q_{\tau}}\left|u_{m}\right|^{\aleph+2} d x d t,
\end{aligned}
$$

где $c^{*} \succ 0$ - постоянная величина.

Используя последний факт и предыдущее неравенство, после несложных оценок получаем справедливость леммы 1.5.

Для краткости изложения подробное доказательство леммы 1.5 и последующей леммы опустим.

ЛЕмма 1.6. Пусть $u_{m}$ удовлетворяет условиям леммы 1.4. Тогда справедливо следующее неравенство:

$$
\begin{aligned}
\left.\left\langle\psi\left(u_{m}\right), C_{2} u_{m t}\right\rangle\right|_{\tau} \geqslant \frac{C_{2}}{2} \int_{Q_{r}}\left|u_{m t}\right|^{2} d x d t+\left.\frac{C_{2}}{p_{1}+2} \int_{\Omega}\left|D u_{m}\right|^{p_{1}+2} d x\right|_{\tau} \\
\quad+\frac{C_{2}}{2} \int_{\Omega}\left|u_{m}\right|^{p_{0}}\left|D u_{m}\right|^{2} d x-\frac{C_{2}}{p_{1}+2} \int_{\Omega}\left|D u_{m}(x, 0)\right|^{p_{1}+2} d x \\
\quad-\left.\frac{C_{2}}{2} \int_{\Omega}\left|u_{m}\right|^{p_{0}}\left|D u_{m}\right|^{2} d x\right|_{t=0}-\frac{p_{0}^{2} C_{2}}{8} \int_{Q_{r}}\left|u_{m}\right|^{2 p_{0}-2} d x d t \\
\quad-\frac{p_{0}^{4} C_{2}^{2}}{16 p_{1}} \int_{Q_{r}}\left|u_{m}\right|^{4 p_{0}-2}\left|D u_{m}\right|^{p_{1}+2} d x d t-\frac{p_{1}}{16} \int_{Q_{r}}\left|D u_{m}\right|^{p_{1}}\left|D^{2} u_{m}\right|^{2} d x d t
\end{aligned}
$$

әде $C_{2}>0$ - некоторая постоянная.

Из лемм 1.3-1.6 получаем справедливость следующей леммы.

ЛЕмма 1.7. Пусть $u_{m}(x, t)$ удовлетворяет условиям леммы $1.4, p_{1} \geqslant 1$, $p_{0} \geqslant 2$. Тогда справедливо следующее неравенство:

$$
\begin{aligned}
\left\langle\psi\left(u_{m}\right)\right. & \left.E\left(u_{m}\right)\right\rangle\left.\right|_{\tau} \geqslant M_{1} \int_{\Omega}\left|u_{m}(x, \tau)\right|^{\left(4 p_{0}-2\right)(\aleph+2)+2} d x \\
& +M_{2} \int_{Q_{r}}|u|^{\left(4 p_{0}-2\right)(\aleph+2)+p_{0}}\left|D u_{m}\right|^{2} d x d t \\
& +M_{3} \int_{Q_{r}}\left|u_{m}\right|^{\left(4 p_{0}-2\right)(\aleph+2)}\left|D u_{m}\right|^{p_{1}+2} d x d t+M_{4} \int_{\Omega}\left|D u_{m}(x, \tau)\right|^{2} d x \\
& +M_{5} \int_{Q_{r}}\left|u_{m}\right|^{p_{0}}\left|D^{2} u_{m}\right|^{2} d x d t+M_{6} \int_{Q_{r}}\left|D u_{m}\right|^{p_{1}}\left|D^{2} u_{m}\right|^{2} d x d t \\
& +M_{7} \int_{\Omega}\left|D u_{m}(x, \tau)\right|^{\aleph+2} d x+M_{8} \int_{Q_{r}}\left|u_{m}\right|^{p_{0}}\left|D u_{m}\right|^{\aleph}\left|D^{2} u_{m}\right|^{2} d x d t \\
& +M_{9} \int_{Q_{r}}\left|D u_{m}\right|^{p_{1}+\aleph}\left|D^{2} u_{m}\right|^{2} d x d t+M_{10} \int_{Q_{r}}\left|u_{m t}\right|^{2} d x d t \\
& +M_{11} \int_{\Omega}\left|D u_{m}(x, \tau)\right|^{p_{1}+2} d x+M_{12} \int_{\Omega}\left|u_{m}(x, \tau)\right|^{p_{0}}\left|D u_{m}(x, \tau)\right|^{2} d x-M_{13},
\end{aligned}
$$


где $M_{i}$ - некоторые положительные постоянные, не зависящие от $u_{m} u$ m.

Отметим,что последнее неравенство получено в результате сложения правых и левых частей неравенств из лемм 1.3-1.6. При этом в правой части получаются отрицательные интегралы вида $\int_{Q_{\tau}}|u|^{\vartheta} d x$ с некоторыми коэффищиентами. Но эти интегралы легко можно оценить интегралом из второго слагаемого в правой части вышеприведенного неравенства с некоторым коэффищиентом, применяя неравенство Пуанкаре (см. лемму 1.7). Следовательно, для получения вышеприведенного неравенства достаточно в лемме 1.3 постоянную $c_{1}$ выбрать сколь угодно большой (см. также определение оператора $\left.E: \Re_{2}(Q) \rightarrow W_{2}^{1}(Q)\right)$.

Далее, оценим сверху второе слагаемое равенства (1.9). Учитьвая, что $f \in$ $L_{2}(0, T ; \stackrel{\circ}{W} \underset{\aleph+2}{1}(\Omega))$, получаем

$$
\begin{aligned}
& \left|\left\langle f, E\left(u_{m}\right)\right\rangle\right|_{\tau}|=|-\int_{Q_{r}} f\left|D u_{m}\right|^{\aleph} D^{2} u_{m} d x d t-\int_{Q_{r}} f D^{2} u_{m} d x d t \\
& \quad+c_{2} \int_{Q_{r}} f u_{m t} d x d t+c_{1} \int_{Q_{r}} f\left|u_{m}\right|^{p_{*}} u_{m} d x d t \mid \\
& \leqslant \frac{c(\varepsilon)}{\aleph+1} \int_{Q_{r}}\left|\frac{\partial f}{\partial x}\right|^{\aleph+2} d x d t+\frac{\varepsilon}{\aleph+1} \int_{Q_{r}}\left|D u_{m}\right|^{\aleph+2} d x d t \\
& \quad+c(\varepsilon) \int_{Q_{r}}\left|\frac{\partial f}{\partial x}\right|^{2} d x d t+\varepsilon \int_{Q_{r}}\left|D u_{m}\right|^{2} d x d t+c(\varepsilon) c_{2} \int_{Q_{r}}|f|^{2} d x d t \\
& \quad+\varepsilon c_{2} \int_{Q_{r}}\left|u_{m t}\right|^{2} d x d t+c_{1} c(\varepsilon) \int_{Q_{r}}|f|^{p_{*}+2} d x d t+c_{1} \varepsilon \int_{Q_{r}}\left|u_{m}\right|^{p_{*}+2} d x d t
\end{aligned}
$$

где $\varepsilon>0$ достаточно мало.

Отметим, что интегралы $\frac{\varepsilon}{\aleph+1} \int_{Q_{\tau}}\left|D u_{m}\right|^{\aleph+2} d x d t$ и $\varepsilon \int_{Q_{\tau}}\left|D u_{m}\right|^{2} d x d t$ легко оцениваются через положительные интегралы из неравенства леммы 1.7 посредством рассуждений, приведенных в доказательстве леммы 1.5.

Таким образом, учитьвая неравенство леммы 1.7 , получаем, что можно найти число $\rho(f)>0$ такое, что

$$
\left\langle\psi\left(u_{m}\right), E\left(u_{m}\right)\right\rangle-\left\langle f, E\left(u_{m}\right)\right\rangle \geqslant 0
$$

или, возвращаясь к первоначальным обозначениям, $\left(\Phi\left(c_{m}\right),\left(c_{m}\right)\right)>0$, т.е. $\Phi$ удовлетворяет всем условиям леммы об остром угле. Таким образом получили, что последовательность $\left\{u_{m}\right\}$ содержится в ограниченном подмножестве пространства $\Re_{2}(Q)$. Проведя рассуждения, аналогичные рассуждениям, проведенным в лемме 1.2 , получим $u_{m} \rightarrow u$ в $\Re_{1}(Q)$.

Далее, учитьвая условия на отображения $E^{-1}$ и $\psi$, можно переходить к пределу в (1.9) при $m \uparrow+\infty$ для каждого $k=1,2, \ldots$ Переходя к пределу в (1.9) при $m \uparrow$ $+\infty$ и произвольном $k \leqslant m$, получаем, что для всякого $k=1,2, \ldots$ выполняется равенство $\left\langle\psi(u), y^{k}\right\rangle=\left\langle f, y^{k}\right\rangle$.

Учитывая полноту системы $\left\{y^{k}\right\}$ в $W_{2}^{1}(Q)$ и замыкая ее в $W_{2}^{1}(Q)$, получаем, что для любого $y \in W_{2}^{1}(Q)$ выполняется равенство $\langle\psi(u), y\rangle=\langle f, y\rangle$. 
Далее, учитывая плотность $W_{2}^{1}(Q)$ в $L_{2}(Q)$ и то, что $\psi(u) \in L_{2}(Q)$, окончательно имеем

$$
\langle\psi(u), y\rangle=\langle f, y\rangle \quad \forall y \in L_{2}(Q)
$$

Теорема 1.1 доказана.

\section{§2. Один дополнительный результат о гладкости}

Далее приведена одна теорема о гладкости решения задачи (1.1)-(1.3), а именно, при некоторых дополнительных условиях доказано, что решения обладают большей гладкостью и содержатся в пространстве

$\Re_{3}(Q)=W_{\infty}^{1}\left(0, T ; L_{2}(\Omega)\right) \cap \Re_{1}(Q) \cap\left\{\left.u|| D u\right|^{\frac{p_{1}}{2}} D u_{t} \in L_{2}(Q),|u|^{\frac{p_{0}}{2}} D u_{t} \in L_{2}(Q)\right\}$.

Иначе говоря, справедлива следующая

ТЕОРемА 2.1. Пусть $p_{0}, p_{1}$ - числа, удовлетворяющие условиям $p_{0} \geqslant 2$, $p_{1} \geqslant 2$. Тогда для любых функиий $u_{0} \in \stackrel{\circ}{W_{r}^{1}}(\Omega)$ u $f \in \stackrel{\circ}{W_{q}^{1}}(Q)$ nри $r=\max \left\{p_{0}+\right.$ $\left.p_{1}+2 ; 2 p_{0}+p_{*}+2 ; \aleph+2\right\}, q=\max \left\{4 ; p_{*}+2 ; \aleph+2\right\}$ решение задачи (1.1)-(1.3) содержится в $\Re_{3}(Q)$.

Сначала приведем некоторые дополнительные объяснения к доказательству теоремы 1.1, точнее, будем пользоваться тем, что доказательство проводится с использованием метода Галёркина. Другими словами, берется полная система функций из соответствующего пространства и находится последовательность приближенных решений. Из этих соображений и из того, как подбираются соответствующие пространства и отображения при доказательстве теоремы 1.1, получаем, что приближенные решения $u_{m}$ данной задачи для любого $m$ содержатся в ограниченном подмножестве пространства $\Re_{2}(Q)$.

Из условий теоремы 2.1 в силу теоремы 1.1 следует, что задача разрешима. Если функция $u(x, t)$, принадлежашая $\Re_{1}(Q)$, является решением этой задачи, то она удовлетворяет уравнению (1.1), точнее, почти всюду выполняется равенство

$$
\frac{\partial u}{\partial t}-D\left(|u|^{p_{0}} D u+|D u|^{p_{1}} D u\right)=f
$$

Теперь, учитьвая то, что правая часть этого равенства содержится в $\stackrel{\circ}{W}{ }_{q}^{1}(Q)$, по крайней мере, после изменения на множестве меры нуль получим, что и левая часть равенства $(2.1)$ содержится в $\stackrel{\circ}{W} \stackrel{1}{q}(Q)$, т.е.

$$
\frac{\partial u}{\partial t}-D\left(|u|^{p_{0}} D u+|D u|^{p_{1}} D u\right) \in \stackrel{\circ}{W_{q}^{1}}(Q) .
$$

Тогда нетрудно видеть, что для любого $v \in L_{2}(Q)$ справедливо следующее равенство:

$$
\int_{Q} \frac{\partial}{\partial t}\left(\frac{\partial u}{\partial t}-D\left(|u|^{p_{0}} D u+|D u|^{p_{1}} D u\right)\right) v d x d t=\int_{Q} v \frac{\partial f}{\partial t} d x d t .
$$


Поскольку $u \in \Re_{1}(Q)$, это равенство для любого $v \in \stackrel{\circ}{W} \underset{2}{1}(Q)$ можно записать следуюшим образом:

$$
\begin{aligned}
\int_{Q} \frac{\partial}{\partial t} & \left(\frac{\partial u}{\partial t}\right) v d x d t-\int_{Q} \frac{\partial}{\partial t}\left(D\left(|D u|^{p_{1}} D u\right)\right) v d x d t \\
& -\int_{Q} \frac{\partial}{\partial t}\left(D\left(|u|^{p_{0}} D u\right)\right) v d x d t=\int_{Q} \frac{\partial f}{\partial t} v d x d t
\end{aligned}
$$

Отсюда получаем, что если последовательность $u_{m}$ является последовательностью приближенных решений задачи (1.1)-(1.3), то при $m \uparrow+\infty$ имеем

$$
\begin{aligned}
\int_{Q} \frac{\partial^{2} u_{m}}{\partial t^{2}} v_{k} d x d t-\int_{Q} \frac{\partial}{\partial t}\left(D\left(\left|D u_{m}\right|^{p_{1}} D u_{m}\right)\right) v_{k} d x d t \\
\quad-\int_{Q} \frac{\partial}{\partial t}\left(D\left(\left|u_{m}\right|^{p_{0}} D u_{m}\right)\right) v_{k} d x d t=\int_{Q} \frac{\partial f}{\partial t} v_{k} d x d t \quad \forall k=1,2, \ldots
\end{aligned}
$$

$\left\{v_{k}(x, t)\right\}_{k=1}^{\infty} \subset \stackrel{\circ}{W} \frac{1}{2}(Q)$ - полная система функций в этом пространстве.

В силу вышеизложенного в этом равенстве, кроме первого слагаемого, остальные интегралы определены для произвольного $v_{k} \in L_{2}(0, T ; \stackrel{\circ}{W} \underset{2}{1}(\Omega))$. Тогда, пользуясь стандартными рассуждениями и тем, что вместо $v(x, t)$ можно брать элемент полной системы, получаем, что первый интеграл также определен для $v \in$ $L_{2}(0, T ; \stackrel{\circ}{W} \underset{2}{1}(\Omega))$.

Далее, так как $\frac{\partial u_{m}}{\partial t} \in L_{2}(0, T ; \stackrel{\circ}{W} \underset{2}{1}(\Omega))$, то из предыдущих объяснений следует, что вместо $v(x, t)$ в уравнение $(2.2)$ можно подставить $\frac{\partial u_{m}}{\partial t}$.

Теперь доказательство теоремы вытекает из следующей леммы.

ЛЕмма 2.1. Пусть выполняются условия теоремы 2.1. Тогда для приближснных решений $u_{m}(x, t)$ справедливы следующие включения:

$$
\begin{gathered}
u_{m t} \in L_{\infty}\left(0, T ; L_{2}(\Omega)\right), \quad\left|u_{m}\right|^{p_{0} / 2} D u_{m t} \in L_{2}(Q), \\
\left|D u_{m}\right|^{p_{1} / 2} D u_{m t} \in L_{2}(Q)
\end{gathered}
$$

Более того, эти выражения содержатся в ограниченных подмножествах соответствующих пространств.

ДокАЗАТЕЛЬСтво. Продифференцируем уравнение (2.1) по $t$ (это возможно в силу приведенных выше объяснений). Имеем

$$
\frac{\partial^{2} u}{\partial t^{2}}-D\left(|u|^{p_{0}} D u_{t}\right)-p_{0} D\left(|u|^{p_{0}-2} u u_{t} D u\right)-\left(p_{1}+1\right) D\left(|D u|^{p_{1}} D u_{t}\right)=f_{t} .
$$

Полученное равенство умножим на $u_{m t}$ и проинтегрируем по $Q_{t}=\Omega \times[0, t)$ :

$$
\begin{aligned}
\int_{Q_{t}} \frac{\partial^{2} u_{m}}{\partial t^{2}} \frac{\partial u_{m}}{\partial t} d x d t-\int_{Q_{t}} D\left(\left|u_{m}\right|^{p_{0}} D u_{m t}\right) u_{m t} d x d t \\
\quad-p_{0} \int_{Q_{t}} D\left(\left|u_{m}\right|^{p_{0}-2} u_{m} u_{m t} D u_{m}\right) u_{m t} d x d t \\
\quad-\left(p_{1}+1\right) \int_{Q_{t}} D\left(\left|D u_{m}\right|^{p_{1}} D u_{m t}\right) u_{m t} d x d t=\int_{Q_{t}} f_{t} u_{m t} d x d t
\end{aligned}
$$


Интегрируя по частям последние три интеграла в левой части предыдушего равенства, получаем

$$
\begin{aligned}
& \frac{1}{2} \int_{Q_{t}} \frac{\partial}{\partial t}\left|u_{m t}\right|^{2} d x d t+\int_{Q_{t}}\left|u_{m}\right|^{p_{0}}\left|D u_{m t}\right|^{2} d x d t \\
& \quad+\left(p_{1}+1\right) \int_{Q_{t}}\left|D u_{m}\right|^{p_{1}}\left|D u_{m t}\right|^{2} d x d t \\
& \quad+p_{0} \int_{Q_{t}}\left|u_{m}\right|^{p_{0}-2} u_{m} u_{m t} D u_{m} D u_{m t} d x d t=\int_{Q_{t}} f_{t} u_{m t} d x d t
\end{aligned}
$$

Используя здесь неравенство Гёльдера и Юнга, получим

$$
\begin{aligned}
&\left.p_{0}\left|\int_{Q_{t}}\right| u_{m}\right|^{p_{0}-2} u_{m} u_{m t} D u_{m} D u_{m t} d x d t \mid \\
& \leqslant\left(p_{0} c\left(\varepsilon_{2}\right)\left\|u_{m}\right\|_{L_{\infty}\left(Q_{t}\right)}^{p_{0}-2}+p_{0} c\left(\varepsilon_{1}\right)\left\|u_{m}\right\|_{L_{\infty}\left(Q_{t}\right)}^{2 p_{0}-2}\right) \int_{Q_{t}}\left|u_{m t}\right|^{2} d x d t \\
& \quad+p_{0} \varepsilon_{2} \int_{Q_{t}}\left|u_{m}\right|^{p_{0}}\left|D u_{m t}\right|^{2} d x d t+p_{0} \varepsilon_{1} \int_{Q_{t}}\left|D u_{m}\right|^{p_{1}}\left|D u_{m t}\right|^{2} d x d t
\end{aligned}
$$

где $\varepsilon_{1}, \varepsilon_{2}$ - достаточно малы.

Далее, используя последнее неравенство, равенство (2.4), а также условие на функцию $f(x, t)$, после несложных оценок получаем справедливость леммы.

ДОКАЗАТЕЛЬСТВо ТЕОРЕМЫ 2.1. Поскольку

$$
\frac{\partial}{\partial t}\left(\left|u_{m}\right|^{p_{0} / 2} D u_{m}\right)=\left|u_{m}\right|^{p_{0} / 2} D u_{m t}+\frac{p_{0}}{2}\left|u_{m}\right|^{p_{0} / 2-2} u_{m} \frac{\partial u_{m}}{\partial t} D u_{m},
$$

то в силу леммы 2.1 и того, что $u_{m} \in \Re_{2}(Q)$, имеем

$$
\frac{\partial}{\partial t}\left(\left|u_{m}\right|^{p_{0} / 2} D u_{m}\right) \in L_{2}(Q) .
$$

Таким образом, $u_{m} \in \Re_{3}(Q)$. Отсюда следует, что $u_{m t}$ ограничено в $L_{\infty}(0, T$; $\left.L_{2}(\Omega)\right)$, a $\left|D u_{m}\right|^{p_{1} / 2} D u_{m t}$ и $\frac{\partial}{\partial t}\left(\left|u_{m}\right|^{p_{0} / 2} D u_{m}\right)$ ограничены в $L_{2}(Q)$.

Следовательно, из последовательности $u_{m}$ можно выбрать подпоследовательность, для которой $u_{m t} \rightarrow u_{t} *$-слабо в $L_{\infty}\left(0, T ; L_{2}(\Omega)\right)($ см. [10]),

$$
\left|D u_{m}\right|^{p_{1} / 2} D u_{m t} \rightarrow \chi \quad \text { слабо в } L_{2}(Q), \quad \frac{\partial}{\partial t}\left(\left|u_{m}\right|^{p_{0} / 2} D u_{m}\right) \rightarrow \eta \quad \text { слабо в } L_{2}(Q) .
$$

Далее, рассуждая так же, как при доказательстве слабой компактности оператора, порожденного задачей (1.1)-(1.3), и пользуясь тем, что $u_{m} \rightarrow u$ в $\Re_{1}(Q)$, имеем

$$
\begin{array}{lll}
\frac{\partial}{\partial t}\left(\left|u_{m}\right|^{p_{0} / 2} D u_{m}\right) \rightarrow \frac{\partial}{\partial t}\left(|u|^{p_{0} / 2} D u\right) & \text { в } \quad L_{2}(Q), \\
\left|D u_{m}\right|^{p_{1} / 2} D u_{m t} \rightarrow|D u|^{p_{1} / 2} D u_{t} & \text { в } \quad L_{2}(Q) .
\end{array}
$$

Таким образом, получаем, что решение задачи содержится в $\Re_{3}(Q)$. Теорема доказана. 


\section{§3. Задача со свободной границей}

Далее исследуется задача

$$
\begin{gathered}
\frac{\partial u}{\partial t}-D\left(|u|^{p_{0}} D u+|D u|^{p_{1}} D u\right)=f(x, t), \quad(x, t) \in Q(T), \\
u\left(s_{1}(t), t\right)=c, \quad t \in[0, T), \\
u(x, 0)=u_{0}(x) \geqslant c>0, \quad x \in\left(s_{1}(0), s_{2}(0)\right),
\end{gathered}
$$

где $s_{i}(t)$ - неизвестные границы области $Q(T)=\left\{(x, t) \in \mathbb{R}^{2} \mid t \in(0, T), s_{1}(t) \leqslant\right.$ $\left.x \leqslant s_{2}(t)\right\}$. Отметим, что $u_{0}(x)$ рассматривается как сужение на $\left(s_{1}(0), s_{2}(0)\right)$ некоторой функции $\tilde{u}_{0}(x)>0$, определенной на отрезке $[a, b] \supset\left[s_{1}(0), s_{2}(0)\right]$, такой, что $D \tilde{u}_{0}\left(s_{1}(0), 0\right)>0, D \tilde{u}_{0}\left(s_{2}(0), 0\right)<0$. (Вместе с тем предполагается, что $\tilde{u}_{0}(x)<c$ при $x \in\left[a, s_{1}(0)\right] \cup\left[s_{2}(0), b\right]$.)

На свободной гранище предполагается выполнение условия типа Стефана (почти всюду)

$$
s_{i}^{\prime}(t)=-\left(c_{1}+c_{2}|D u|^{p_{1}}\right) D u\left(s_{i}(t), t\right), \quad i=1,2,
$$

где $c_{1}=c^{p_{0}-1}, c_{2}=1 / c$. Кроме того, предположим, что

$$
u(x, t)=0 \text { при } x \prec s_{1}(t), \quad x \succ s_{2}(t), \quad t \in[0, T),
$$

и что supp $f \subset Q(T)$.

Допустим теперь, что существует функция $u(x, t)$, почти всюду удовлетворяюшая условиям (3.1)-(3.5). Тогда полученное решение можно рассматривать как решение уравнения с разрьвными коэффищиентами, точнее говоря, как решение следуюшего уравнения:

$$
\frac{\partial u}{\partial t}-D\left(a_{1}(x, t)|u|^{p_{0}} D u+a_{2}(x, t)|D u|^{p_{1}} D u\right)=f
$$

где

$$
a_{i}(x, t)=\left\{\begin{array}{l}
1 \quad \forall(x, t) \in Q_{T}, \\
0 \quad \forall(x, t) \in\left\{(x, t) \mid t \in[0, T], \quad x<s_{1}(t) \vee x>s_{2}(t)\right\} .
\end{array}\right.
$$

Как известно из общей теории квазилинейных уравнений, в дивергентном случае краевые задачи устойчивы по отношению к вариациям коэффициентов и правых частей уравнений (см. [11]). Поэтому решения дифракционных задач, к которым относится и задача с уравнением $\left(3.1^{\prime}\right)$ и краевыми условиями, вытекающими из (3.2), (3.3), можно получать как пределы хороших решений уравнений со сглаженными коэффищиентами $a_{1 m}$ и $a_{2 m}\left(a_{1 m} \rightarrow a_{1}, a_{2 m} \rightarrow a_{2}\right)$ и правыми частями $f_{m}$, аппроксимирующими функцию $f$ (см. [11]). Используя это, проведем вьвод условия (3.4) согласно схеме, изложенной в [11]. Для решений уравнений типа $\left(3.1^{\prime}\right)$ со сглаженными коэфффищиентами $a_{1 m}$ и $a_{2 m}$ эквивалентны равенства

$$
\begin{aligned}
& \int_{Q} \frac{\partial u}{\partial t} \varphi d x d t-\int_{Q} D\left(a_{1 m}|u|^{p_{0}} D u+a_{2 m}|D u|^{p_{1}} D u\right) \varphi d x d t=\int_{Q} f_{m} \varphi d x d t \\
& -\int_{Q} u \frac{\partial \varphi}{\partial t} d x d t+\int_{Q}\left(a_{1 m}|u|^{p_{0}} D u+a_{2 m}|D u|^{p_{1}} D u\right) D \varphi d x d t=\int_{Q} f_{m} \varphi d x d t
\end{aligned}
$$


где $\varphi$ такое, что $\operatorname{supp} \varphi \subset Q=[a, b] \times[0, T], \varphi \in \stackrel{\circ}{C^{\infty}}(Q)$.

Итак, используя вышеизложенное, не умоляя обшности, можно считать, что рассмотренная задача является пределом краевой задачи для уравнения с гладкими коэффициентами и правой частью. Учитывая это, получаем, что отмеченные эквивалентные равенства справедливы и для уравнения $\left(3.1^{\prime}\right)$. Отсюда вытекает, что

$$
\begin{gathered}
\int_{0}^{T} \int_{s_{1}(t)}^{s_{2}(t)} \frac{\partial u}{\partial t} \varphi d x d t-\int_{0}^{T} \int_{s_{1}(t)}^{s_{2}(t)} D\left(|u|^{p_{0}} D u+|D u|^{p_{1}} D u\right) \varphi d x d t \\
=\int_{0}^{T} \int_{s_{1}(t)}^{s_{2}(t)} f \varphi d x d t \\
-\int_{0}^{T} \int_{s_{1}(t)}^{s_{2}(t)} u \frac{\partial \varphi}{\partial t} d x d t+\int_{0}^{T} \int_{s_{1}(t)}^{s_{2}(t)}\left(|u|^{p_{0}} D u+|D u|^{p_{1}} D u\right) D \varphi d x d t \\
=\int_{0}^{T} \int_{s_{1}(t)}^{s_{2}(t)} f \varphi d x d t .
\end{gathered}
$$

Далее, преобразуя уравнение $\left(3.1^{\prime \prime}\right)$, получим

$$
\begin{aligned}
& -\int_{0}^{T} \int_{s_{1}(t)}^{s_{2}(t)} u \frac{\partial \varphi}{\partial t} d x d t+\int_{0}^{T} \int_{s_{1}(t)}^{s_{2}(t)}\left(|u|^{p_{0}} D u+|D u|^{p_{1}} D u\right) D \varphi d x d t \\
& \quad+\int_{0}^{T} \int_{s_{1}(t)}^{s_{2}(t)} \frac{\partial}{\partial t}(u \varphi) d x d t \\
& \quad-\int_{0}^{T} \int_{s_{1}(t)}^{s_{2}(t)} D\left(\left(|u|^{p_{0}} D u+|D u|^{p_{1}} D u\right) \varphi\right) d x d t=\int_{0}^{T} \int_{s_{1}(t)}^{s_{2}(t)} f \varphi d x d t .
\end{aligned}
$$

Последнее равенство равносильно следующему:

$$
\begin{aligned}
-\int_{0}^{T} \int_{s_{1}(t)}^{s_{2}(t)} u \frac{\partial \varphi}{\partial t} d x d t+\int_{0}^{T} \int_{s_{1}(t)}^{s_{2}(t)}\left(|u|^{p_{0}} D u+|D u|^{p_{1}} D u\right) D \varphi d x d t \\
\quad-\int_{0}^{T}\left(\left.|u|^{p_{0}} D u \varphi\right|_{s_{2}(t)}-\left.|u|^{p_{0}} D u \varphi\right|_{s_{1}(t)}+\left.|D u|^{p_{1}} D u \varphi\right|_{s_{2}(t)}\right. \\
\left.\quad-\left.|D u|^{p_{1}} D u \varphi\right|_{s_{1}(t)}\right) d t+\int_{0}^{T} \frac{\partial}{\partial t}\left(\int_{s_{1}(t)}^{s_{2}(t)} \varphi u d x\right) d t \\
\quad-\int_{0}^{T} s_{2}^{\prime}(t) u\left(s_{2}(t), t\right) \varphi\left(s_{2}(t), t\right) d t+\int_{0}^{T} s_{1}^{\prime}(t) u\left(s_{1}(t), t\right) \varphi\left(s_{1}(t), t\right) d t \\
=\int_{0}^{T} \int_{s_{1}(t)}^{s_{2}(t)} f \varphi d x d t .
\end{aligned}
$$

Теперь, учитывая, что supp $\varphi \subset Q$, в силу уравнения $\left(3.1^{\prime \prime \prime}\right)$ и произвольности функции $\varphi$ получаем

$$
\left.\int_{0}^{T}\left(|u|^{p_{0}}+|D u|^{p_{1}}\right) D u \varphi\right|_{s_{i}(t)} d t=-\int_{0}^{T} s_{i}^{\prime}(t) u\left(s_{i}(t), t\right) \varphi\left(s_{i}(t), t\right) d t .
$$


Если функция $D u(x, t)$ непрерывна, то почти всюду выполняется равенство

$$
s_{i}^{\prime}(t) u\left(s_{i}(t), t\right)=-\left(|u|^{p_{0}}+|D u|^{p_{1}}\right) D u\left(s_{i}(t), t\right) .
$$

Это равенство есть не что иное, как условие (3.4).

Итак, остается показать существование достаточно гладкого решения $u(x, t)$ уравнения (1.1) такого, что вместе с кривыми $s_{i}(t)$, определенными по $(3.2),(3.4)$, тройка $\left\{u(x, t), s_{1}(t), s_{2}(t)\right\}$ удовлетворяла бы условиям (3.1)-(3.5).

Для этого рассмотрим следуюшую задачу:

$$
\begin{aligned}
& \frac{\partial u}{\partial t}-D\left(|u|^{p_{0}} D u+|D u|^{p_{1}} D u\right)=f(x, t), \quad(x, t) \in Q, \\
& u(x, 0)=\tilde{u}_{0}(x)>0, \quad x \in(a, b), \\
& \left.u\right|_{\Gamma}=0, \quad \Gamma=\partial \Omega \times[0, T)
\end{aligned}
$$

(как уже отмечалось, $u_{0}(x)$ является сужением некоторой функции $u(x, 0)=$ $\tilde{u}_{0}(x)>0$, определенной на отрезке $\left.[a, b]\right)$.

В предыдущих параграфах было доказано существование определенного гладкого решения задачи (3.6)-(3.8). Однако для дальнейших исследований необходимо доказать следующую лемму.

ЛЕмма 3.1. Пусть выполнены условия теоремы 2.1. Тогда для решения задачи (3.6)-(3.8) справедливы следующие включения:

$$
\begin{aligned}
u & \in C^{0}\left(0, T ; C^{\gamma}(\Omega)\right), & & 0<\gamma<1, \\
D u & \in C^{0}\left(0, T ; C^{\delta}(\Omega)\right), & & 0<\delta<1 .
\end{aligned}
$$

Предварительно докажем следуюшие два утверждения.

УТВЕРЖДЕНИЕ 3.1. Пусть $u(x, t) \in \Re_{3}(Q)$ - решение задачи (3.6)-(3.8) при условиях теоремы 2.1. Тогда справедливо следующее включение: $|D u|^{p_{1}} D u \in$ $L_{\infty}\left(0, T ; W_{2}^{1}(\Omega)\right)$.

ДокАЗАТЕЛЬСтво. Перепишем уравнение (3.6) в следуюшем виде:

$$
\left(p_{1}+1\right)|D u|^{p_{1}} D^{2} u+|u|^{p_{0}} D^{2} u=f-u_{t}-p_{0}|u|^{p_{0}-2} u|D u|^{2} .
$$

Из результатов предыдуших параграфов и условия на функцию $f(x, t)$ имеем

$$
\left(p_{1}+1\right)|D u|^{p_{1}} D^{2} u+|u|^{p_{0}} D^{2} u=f-u_{t}-p_{0}|u|^{p_{0}-2} u|D u|^{2} \in L_{\infty}\left(0, T ; L_{2}(\Omega)\right) .
$$

Следовательно, $|D u|^{p_{1}} D^{2} u \in L_{\infty}\left(0, T ; L_{2}(Q)\right)$. Учитывая, что

$$
|D u|^{p_{1}} D u \in L_{\infty}\left(0, T ; L_{2}(Q)\right)
$$

(см. определение $\left.\Re_{1}(Q)\right)$, получаем справедливость утверждения. 
УТВЕРЖДЕНИЕ 3.2. В условиях леммы 3.1 справедливо включение $и \in$ $C\left(\Omega ; C^{\frac{1}{2}}(0, T)\right)$.

ДокАЗАТЕЛЬСТво. Поскольку $u \in \Re_{3}(Q)$, то, в частности,

$$
|u|^{p_{0} / 2} u \in L_{2}\left(\Omega ; W_{2}^{1}(0, T)\right), \quad \frac{\partial}{\partial x}\left(|u|^{p_{0} / 2} u\right) \in L_{2}\left(\Omega ; W_{2}^{1}(0, T)\right) .
$$

Отсюда на основании известной леммы (см. [10]) имеем

$$
|u|^{p_{0} / 2} u \in C\left(\Omega ; W_{2}^{1}(0, T)\right)
$$

Далее, используя теорему Кондрашова (см. [12]), получим, что

$$
C\left(\Omega ; W_{2}^{1}(0, T)\right) \subset C\left(\Omega ; C^{1 / 2}(0, T)\right),
$$

т.е. $|u|^{p_{0} / 2} u \in C\left(\Omega ; C^{1 / 2}(0, T)\right)$.

Ввиду того, что

$$
\left|u\left(x, t_{2}\right)-u\left(x, t_{1}\right)\right|^{\frac{p_{0}}{2}+1} \leqslant\left. l_{1}|| u\right|^{\frac{p_{0}}{2}} u\left(x, t_{2}\right)-|u|^{\frac{p_{0}}{2}} u\left(x, t_{1}\right)\left|\leqslant l_{2}\right| t_{2}-\left.t_{1}\right|^{\frac{1}{2}},
$$

имеем

$$
\left|u\left(x, t_{2}\right)-u\left(x, t_{1}\right)\right| \leqslant l_{3}\left|t_{2}-t_{1}\right|^{\frac{1}{p_{0}+2}} \quad \forall t_{2}, t_{1} \in[0, T], \quad x \in \Omega .
$$

Следовательно, $u(x, t) \in C\left(\Omega ; C^{1 / 2}(0, T)\right)$. Тем самым, утверждение доказано.

Отметим, что поскольку $|D u|^{p_{1}} D u \in L_{\infty}\left(0, T ; W_{2}^{1}(\Omega)\right) \cap W_{2}^{1}\left(0, T ; L_{2}(\Omega)\right)$ (см. утверждение 3.1 и определение $\Re_{3}(Q)$ ), то из теоремы Кондрашова следует, что $D u \in L_{\infty}\left(0, T ; C^{\frac{1}{2\left(p_{1}+1\right)}}(\Omega)\right)$.

Кроме того, известна следующая лемма.

Лемма 3.2 [11]. Пусть $\Omega \subset \Re^{n}$ удовлетворяет условию конуса, функиия $u(x, t)$ в $Q=\Omega \times(0, T)$ удовлетворяет условию Гёльдера по $t$ с показателем $\alpha$ и константой Гёльдера $\mu_{1}$ и имеет производные $D_{i} u$, которые при любом $t$ из $[0, T]$ удовлетворяют условию Гёльдера по $x$ с показателем $\beta$ и константой Гёльдера $\mu_{2}$. Тогда производнье $D_{i}$ и удовлетворяют в $Q$ условию Гёльдера nо $t$ с показателем $\tilde{\delta}=\alpha \beta /(1+\beta)$ и константой Гёльдера $\tilde{\mu}$, определяемой лишь $\alpha, \beta, \mu_{1}, \mu_{2}, d$ и телесным углом при вершине конуса.

ДоКАЗАТЕЛЬСТво. Утверждение леммы остается в силе и в том случае, если потребовать, чтобы производные $D_{i} u$ удовлетворяли условию Гёльдерапри почти всех $t$, с той лишь разнищей, что тогда $D_{i} u$ удовлетворяют в $Q$ условию Гёльдера после соответствующего доопределения этих функций на множестве меры нуль.

Учитывая все вышеизложенное, в силу утверждения 3.2 и последней леммы получаем, что $D u \in C\left(0, T ; C^{\delta}(\Omega)\right)$. Лемма доказана.

В силу выбора $\tilde{u}_{0}(x)$, а точнее, в силу того, что $D \tilde{u}_{0}\left(s_{i}(0), 0\right) \neq 0$, учитывая найденную в лемме 3.1 гладкость, получим на основании теоремы о неявной функции сушествование некоторых локально единственных кривых $s_{i}(t)$, исходяших из 
точек $\left(s_{i}(0), 0\right)$, на которых выполняется условие $u(x, t)=c$. Нетрудно заметить тогда, что сужение на $Q(T)$ решения задачи (3.6)-(3.8) является искомым решением задачи (3.1)-(3.5).

Поскольку $D u\left(s_{1}(0), 0\right)>0$ и $D u\left(s_{2}(0), 0\right)<0$, получаем, что в силу условия (3.4) эти кривые соответственно локально неубывающая и невозрастающая до некоторой точки $\left(x_{i}, t_{i}\right)$, в которой $D u=0$ (если такая точка найдется). Тогда справедлива следующая

ЛЕмма 3.3. В условиях теоремы 2.1 существуют точки $\left(x\left(t_{1}\right), t_{1}\right),\left(x\left(t_{2}\right)\right.$, $\left.t_{2}\right)$, до которых функции $s_{1}(t), s_{2}(t)$ являются неубывающей и невозрастающей соответственно.

Итак, пусть $D u\left(x_{i}, t_{i}\right)=0, i=1,2$. Тогда, например, из точки $\left(x_{1}, t_{1}\right)$ может выйти сколь угодно много кривых, на которых $u(x, t)=c$. В силу непрерывности функции $u(x, t)$ эти кривые являются непрерьвными. Выберем из этих кривых самую крайнюю при движении по часовой стрелке вокруг точки $\left(x_{1}, t_{1}\right)$. Возможны следуюшие два предположения:

1) какую бы точку $(\tilde{x}, \tilde{t})$ кривой мы не взяли, продолжение ее будет лежать вьше прямой $t=\tilde{t}$ (рис.1);

2) предположение 1) неверно.

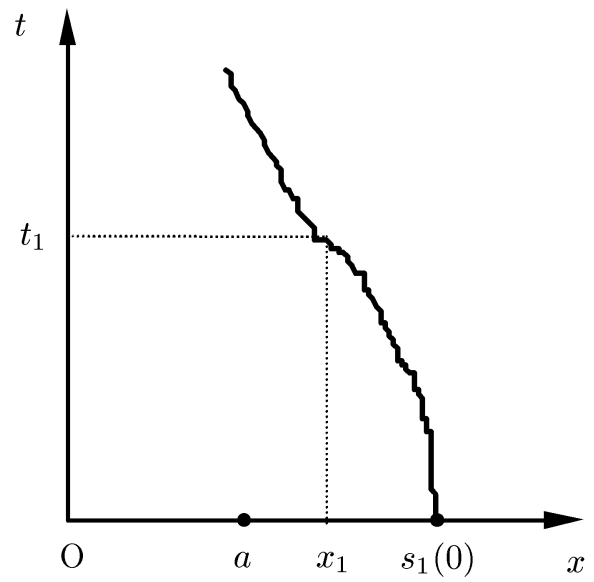

Рис. 1

Рассмотрим далее оба предположения.

1) Полученную после продолжения кривую вновь обозначим через $s_{1}(t)$. Поскольку $D u$ везде удовлетворяет условию (3.10), то ясно, что во всех точках этой части кривой $D u$ определено. Заметим сразу же, что $D u$ на продолженной кривой $s_{1}(t)$ также не может быть меньше нуля в силу того, что справа от $s_{1}(t)$ имеет место условие $u>c$ и $u\left(s_{1}(t), t\right)=c$.

Исходя из этого, докажем, что кривая $s_{1}(t)$ не возрастает.

Предположим обратное, т.е. допустим, что на некотором отрезке $\left[t^{\prime}, t^{\prime \prime}\right]$ функция $s_{1}(t)$ возрастает. Тогда на этом отрезке в силу теоремы Лебега [13] функция $s_{1}(t)$ 
имеет почти всюду производную. Указанная производная будет всюду не меньше нуля. С другой стороны, так как $D u\left(s_{1}(t), t\right) \geqslant 0$, то $s_{1}^{\prime}(t) \leqslant 0$ в силу условия (3.4). Следовательно, $s_{1}^{\prime}(t)=0$ почти всюду на $\left[t^{\prime}, t^{\prime \prime}\right]$.

Известно (см. [13]), что если производная монотонной абсолютно непрерывной функции равна нулю почти всюду, то эта функция постоянная. Таким образом, если $s_{1}(t)$ - абсолютно непрерывная функция, то указанная теорема приходит в противоречие с предположением о том, что $s_{1}(t)$ - возрастающая функция. Иначе говоря, если мы докажем, что $s_{1}(t)$ абсолютно непрерывна, то отсюда будет следовать, что не существует такого интервала $\left[t^{\prime}, t^{\prime \prime}\right]$, где $s_{1}(t)$ возрастает.

Известно, что если для двух суммируемых функций $v_{1}$ и $v_{2}$ выполнено равенство $v_{1}^{\prime}=v_{2}^{\prime}$ в обобщенном смысле, то $v_{1}-v_{2}=$ const (почти всюду). Пусть $v_{1}=s_{1}$, а $v_{2}$ - функция, восстановленная по производной $v_{2}^{\prime}=\left(|D u|^{p_{1}} D u\right)\left(s_{1}(t), t\right)$. Так как $|D u|^{p_{1}} D u$ - непрерывная функция, то функция $v_{2}$ абсолютно непрерывна. Поскольку $v_{1}-v_{2}=$ const почти всюду, то это равенство выполнено всюду, так как $s_{1}$ и $v_{2}$ - непрерывные функции. Но $v_{2}$ абсолютно непрерывна. Следовательно, $s_{1}=$ const $+v_{2}$ абсолютно непрерывная. Таким образом, получили, что $s_{1}(t)$ не может быть возрастающей функцией.

Итак, функция $s_{1}(t)$ невозрастающая.

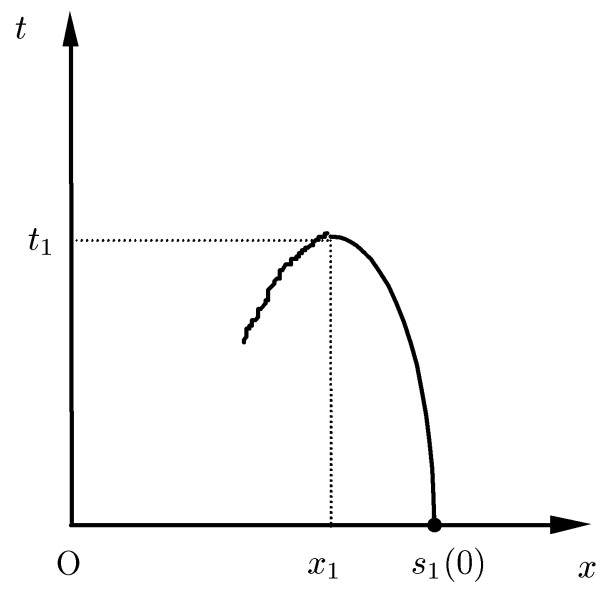

Рис. 2.

2) Допустим теперь, что в точке $\left(x_{1}, t_{1}\right)$ предположение 1$)$ нарушается (рис. 2). Продолжение кривой $s_{1}(t)$ из точки $\left(x_{1}, t_{1}\right)$ обозначим также через $s_{1}(t)$. Поскольку в окрестности точки $\left(x_{1}, t_{1}\right)$, слева от продолжения кривой $s_{1}(t)$ выполняется неравенство $u(x, t)>c$, то на этой части кривой справедливо неравенство $D u\left(s_{1}(t), t\right) \leqslant 0$. Отсюда, вновь применяя условие (3.4) и рассуждения, аналогичные приведенным выше, получаем, что $s_{1}(t)$ - неубываюшая функция начиная с некоторого $t^{\prime}<t_{1}$.

Рассмотрим дальнейшее поведение $s_{1}(t)$. Естественно, что $s_{1}(t)$ может пересекать ось О $x$ только в точке $\left(s_{1}(0), 0\right)$, так как $\tilde{u}_{0}<c$ на интервале $\left[a, s_{1}(0)\right)$. Но и 
этого не может произойти, поскольку из точки $\left(s_{1}(0), 0\right)$ исходит лишь одна кривая. Поэтому функция $s_{1}(t)$, имея конечное или бесконечное число "пиковых точек" типа точки $\left(x_{1}, t_{1}\right)$, в случае 2$)$, показанном на рис. 2 , будет убывать.

Такие же рассуждения, примененные к точке $\left(s_{1}(0), 0\right)$, можно использовать для доказательства того факта, что из точки $\left(s_{2}(0), 0\right)$ выходит некоторая непрерьвная кривая, имеюшая почти всюду производную. Но в отличие от кривой $s_{1}(t)$, кривая, исходяшая из точки $\left(s_{2}(0), 0\right)$, не убывает в какой-то окрестности указанной точки.

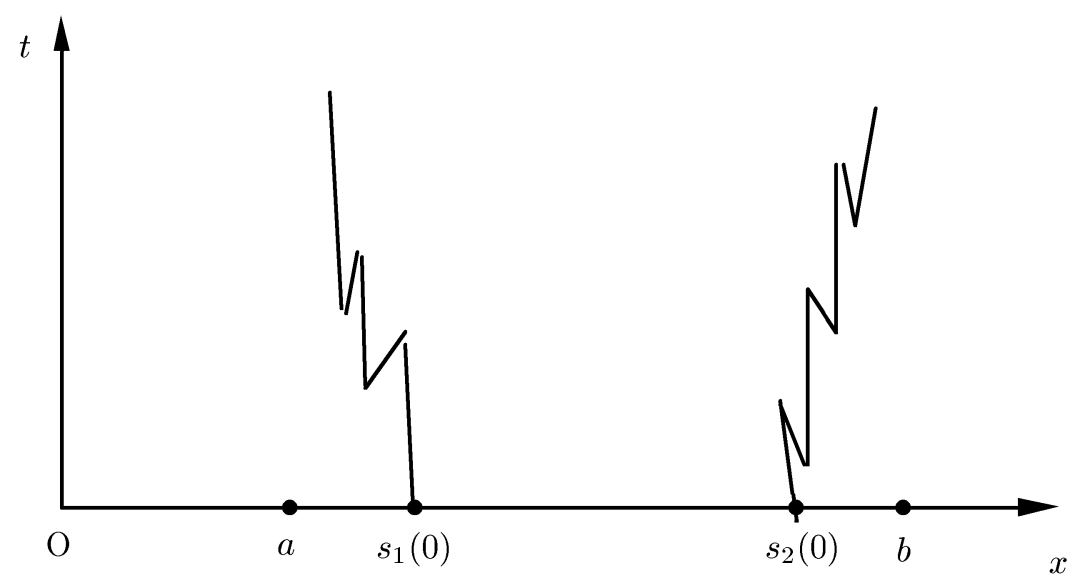

Рис. 3

Далее, кривая $s_{2}(t)$, возможно, образуя "пиковые" точки, отдаляется от кривой $s_{1}(t)$ (рис. 3.).

Таким образом, верна следующая

ЛЕмма 3.4. Кривая $s_{1}(t),\left(s_{2}(t)\right)$ - глобально невозрастающая (неубьвающая). Однако $s_{1}(t)\left(s_{2}(t)\right)$ локально может содержать участки возрастания (убивания), не пересекающиеся с прямой $t=0$.

Из проведенных рассуждений вытекает справедливость следуюшей теоремы.

TЕорема 3.1. Пусть $p_{0} \geqslant 2, \quad p_{1} \geqslant 2$. Тогда для любых функиий $f \in$ $\stackrel{\circ}{W}{ }_{q}^{1}(Q(T))$ u $\tilde{u}_{0} \in \stackrel{\circ}{W}_{r}^{1}(\Omega)$ npu $r=\max \left\{p_{0}+p_{1}+2 ; 2 p_{0}+p_{*}+2, \aleph+2\right\}, \quad q=$ $\max \left\{4, p_{*}+2, \aleph+2\right\}$ задача (3.1)-(3.5) разрешима в $\Re_{3}(Q) \times W_{2}^{1}(0, T) \times W_{2}^{1}(0, T)$, m.e. $s_{i}(t) \in W_{2}^{1}(0, T), u \in \Re_{3}(Q(T))$.

\section{Список литературы}

1. Данилюк И. И. О задаче Стефана // УМН. 1985. Т. 40. № 5. С. 245.

2. Фридман А. Вариационные принципы и задачи со свободными границами. М.: Наука, 1990.

3. Солтанов K. H. Теоремы вложения для нелинейных пространств и разрешимость некоторых нелинейных некоэрцитивных уравнений. Деп. в ВИНИТИ 16.09.91, № 3697-В 91. 
4. Новрузов $Э$. Б. О гладкой разрешимости уравнений типа уравнений неустойчивой фильтрации // Тр. Ин-та мат. и мех. 1996. Т. 6. С. 179-183.

5. Солтанов K. H. О нелинейных уравнениях вида $F(x, u, D u, \Delta u)=0 / /$ Матем. сб. 1993. Т. 184. №11. С. 131-160.

6. Солтанов K.H. Существование и несуществование глобального решения некоторых эллиптико-параболических уравнений // Дифф. уравн. 1993. Т. 29. № 4. С. 646-661.

7. Soltanov K. N., Sprekels J. Nonlinear equations in nonreflexive Banach spaces and fully Nonlinear equations // Adv. in Math. Sci. and Appl. 1999. V. 9. № 2. P. 939-972.

8. Солтанов $K . H$. Некоторые теоремы вложения и их приложения к нелинейным уравнениям // Дифф. уравн. 1984. Т. 20. № 12. С. 2181-2184.

9. Вишик М. И. О разрешимости краевых задач для квазилинейных параболических уравнений высших порядков // Матем. сб. 1962. Т. 59. № 7. С. 289-325.

10. Лионс ЖК. Л. Некоторые методы решения нелинейных краевых задач. М.: Мир, 1972.

11. Ладыжсенская О.А., Солонников В. А., Уральчева Н. Н. Линейные и квазилинейные уравнения параболического типа. М.: Наука, 1967.

12. Гилбарг Д., Трудингер Н. Эллиптические дифференциальные уравнения с частными производными второго порядка. М.: Наука, 1989.

13. Колмогоров A.H., Фомин C. В. Элементы теории функций и функционального анализа. М.: Наука, 1972.

E-mail: soltanov@dcacs.ab.az

Поступило в редакцию

soltanov@lan.ab.az

26.XI. 1999

ksoltanov@hotmail.com 